\title{
SIMULATION MODEL FOR HARBOR VERIFICATION AND MANAGMENT
}

\author{
Sebastián Solari $^{1,2}$, Antonio Moñino ${ }^{1}$, Asunción Baquerizo ${ }^{1}$, Miguel A. Losada ${ }^{1}$
}

This work presents an integrated model for harbor verification and management. The model is based on simulation techniques and includes a queuing system for waiting time calculations and estimates failure probabilities. Within the model, climatic agents (wave, currents, etc.) are transformed in order to know their values at any subset of the system, and the response of the ship to the different agents is computed, in order to estimate failure probability during transits. The model is applied to a case study, at the Bay of Cádiz Harbor, Spain, and some example applications are show.

Keywords: Harbor simulation; Level III verification; Harbor management

\section{INTRODUCTION}

Harbor terminals are complex systems, subject to the action of several climate and usage and exploitation agents, which must fulfil overall safety and operationality criteria (Losada, 2001).

Surveyed harbor simulation models focus either on operational features, estimating the capacity and performance of harbor terminals by studying use and exploitation agents, or on safety factors, studying the climate agents and ship behaviour.

Quy et al. (2008) develop a model to evaluate safety and operationality, as well as some aspects of the performance of a bulk terminal. According to them most existing models focus on the capacity of the terminals, but do not include safety during transits and operations (e.g. Shabayek and Yeung, 2001; Bierwirth and Meisel, 2009).

On the other hand, models that place an emphasis on safety during transit and operations impose a maximum acceptable failure probability for each transit or operation (e.g.: Spencer et al. 1990; Quy et al. 2008). However, in general, they do not estimate the overall failure probability during the entire useful life of the infrastructure. Moreover, many of those models do not make a further study of safety effects on the operationality and service levels of the terminals (e.g. Spencer et al. 1990; Briggs et al. 2003).

For navigation areas, safety, operationality and performance of the system are interrelated indeed, the probability of occurrence of a failure related with ships in transit increases with the number of ships served. On the basis of this fact, a simulation model that takes into account safety, operationality and capacity of the navigation areas and docks at the same time is proposed. This model is intended to: (a) verify with level III techniques both safety and operationality of the navigation areas of the harbors, and (b) manage navigation areas during their useful life.

The model is applied to a new container terminal in the Bay of Cadiz Harbor in southern Spain.

The article is organized as follows. First, the definition of the general methodology is introduced. Then, its implementation to a case study is presented. Finally, some examples of the model applicability are discussed, followed by a summary of the main conclusions derived from this work.

\section{MODEL DESCRIPTION}

The model simulates the performance of the harbor with time using two main modules. One for the track of the ships that registers their positions and waiting times. A second module calculates the value of the climatic agents on each subset of the system, as well as the actions exerted by these agents on the ships. Figure 1 shows schematically the global functioning of the proposed model.

The model requires as inputs time series of climatic agents and of use and exploitation agents and provides time series of several indices used to assess the performance of the system.

Input series of climatic agents are waves on deep waters, winds and astronomic tide levels on the harbor. These agents are transformed inside the model in order to know its value on each subset of the systems (e.g.: wave propagation) and to know the value of other agents (e.g.: estimation of the meteorological tide and the currents).

The ships are the only use and exploitation agent of the navigation areas included in the model. Input to the model are time series of ships calling to harbor, along with their characteristics and cargo.

Under certain pre-established usage policy, and using the values of the climatic agents, the model assesses the operational condition of the navigation areas, that can be operative or non-operative, and

\footnotetext{
${ }^{1}$ Grupo de Dinámica de Flujos Ambientales, Universidad de Granada, Av. del Mediterráneo s/n edif. CEAMA, Granada, 18006, Spain

2 Instituto de Mecánica de los Fluidos e Ingeniería Ambiental, Universidad de la República, Julio Herrera y Reissig 565, Montevideo 11300, Uruguay
} 
the availability of docks. Accordingly it allows or not the entrance of the ship that otherwise remains in the entrance queue.

When entrance transit is allowed, the model tracks the position of the ship during transit, estimating the failure probability on each subset of the entrance channel and on the manoeuvring area, as well as the overall failure probability during the complete transit.

Once it arrives to the docks, the ship remains there during a period of time that depends mainly on the total volume of cargo to be loaded and/or unloaded. Dry areas of the harbour are not included in the model, so a mean capacity for loading/unloading must be assumed. This capacity, along with the total volume to be moved, defines the duration of the stay of the ship at the docks.

The exit transit begins once the ships finish the load/unload stage. The exit stage is similar to that described for the entrance transit and consists of the following steps: verification of operational state of the navigation areas, registration of the exit waiting time, and estimation of the failure probability during exit transit.

Once the simulation is finished, the model gives the failure probability on each subset of the navigation areas, the waiting time for each ship for entrance and for exit, time series of the operational states of the navigation areas, and of the occupation level of the docks. Using these outputs it is possible to construct the probability distribution functions that describe the responds of the system.

The performance of the system in relation to the quality of the service is assessed through the waiting times. For this, the expected and maximum waiting times are estimated, as well as the waiting time probability density function.

In regards with the operationality and safety of the navigation areas during its useful life, each simulation gives only one possible sample of these variables. For estimating the expected operationality and safety, and they PDF, several simulation of the useful life must be run. For each simulation, new time series of the agents must be randomly generated.

The methodology used for the definition of the subsets of the navigation areas, the time step of the simulation and the estimation of the failure probability, are described on next section. Specific aspects related with the implementation of the model are described on Implementation section.

\section{METHODOLOGY}

The estimation of the failure probability during transits is based on two main concepts that are briefly discussed next: subsets of the navigation area and transit states. A more in deep description of these concepts can be found in Losada et al. (2009).

\section{Channel subsets definition}

Navigation areas are divided on subsets. On each subset, the level of the actions exerted on the ship by the agents can be assumed to be uniform. For the case of the failure modes of a ship in transit ("bottom hitting" and "exceedance of the channel way marks"), the following agents must be uniform in each subset: the wave height, period and direction, the speed and the direction of the currents, the still water level and the seabed depth, and the speed and the direction of the ship. Wind is assumed uniform on the navigation areas and is not taken into account on the definition of the subsets.

\section{Definition of agents state}

For the application of the verification equations that are used to estimate the failure probability, it is necessary to identify a characteristic time for which the level of the actions exerted on the ship is statistically stationary. In a similar way to what has been done for subsets definition, the definition of the characteristic time is done in terms of the agents.

Each agent included in the model has its own characteristic time, or state length, for which the agent can be assumed stationary or statistically stationary. For the waves and the winds, this is commonly taken as one to three hours. Tide amplitude is stationary on a semidiurnal or diurnal scale of time. Depending on this amplitude, the sea level can be assumed constant on a time of scales $\mathrm{O}$ (minutes) to $\mathrm{O}$ (hours).

The characteristic time used for the definition of the climatic state $\left(\Delta T_{\text {state }}\right)$, is the minimum of all the characteristic times involved $\left(\Delta T_{\text {state }_{-} i}\right)$. 


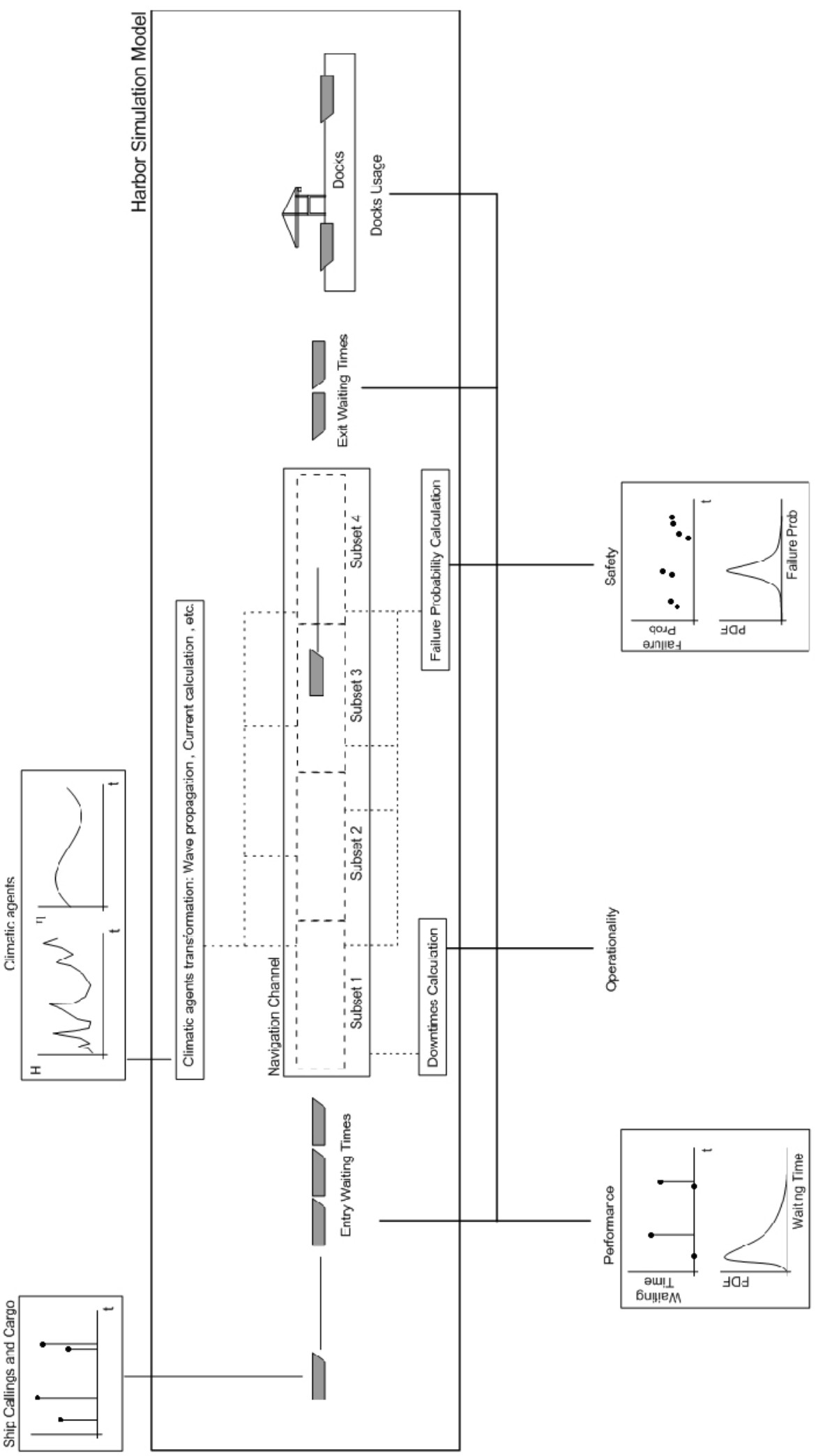

Figure 1. Outline of the simulation model. 


\section{Transit state definition}

The transit state is the unit of time for which the failure probability is estimated. During a transit state, the ship is travelling in a subset (i.e.: under uniform conditions), and all agents can be assumed as stationary or statistically stationary.

The length of a transit state $\left(\Delta T_{\text {transit }}\right)$ is the minimum time step needed by: (a) one of the agents to change its state $\left(\Delta T_{\text {state }}\right)$, or (b) the ship to leave the current subset $\left(\Delta T_{\text {subset }}\right)$ :

$$
\Delta T_{\text {transit }}=\min \left(\Delta T_{\text {subset }}, \Delta T_{\text {state }}\right)
$$

In order to know the time needed by the ship to leave the current subset, the model must track the position of the ships on time.

\section{Failure probability calculation}

With the transit states defined in the way previously described, it is possible to estimate the failure probability during a transit state $i\left(P_{f_{-} i}\right)$ using an equation of state, which depends on the failure mode involved.

Knowing the failure probability for each one of the transit states that makes up a transit, it is possible to estimate the overall failure probability during transit:

$$
P_{f_{-} \text {transit }}=1-\prod_{i=1}^{N_{i}}\left(1-P_{f_{-} i}\right)
$$

where $N_{i}$ is the number of transit states that make up the transit.

The overall failure probability during the useful life of the navigation areas can be estimated as:

$$
P_{f_{-} \text {channel }}=1-\prod_{j=1}^{N_{j}}\left(1-P_{f_{-} \text {transit_ } j}\right)
$$

where it has been assumed that there exists $N_{j}$ non simultaneous transits on the useful life of the navigation areas, including both the entrance and exit transits.

Special attention must be paid to the dependence among transits states that is inherit from the time dependence of the climatic agents. Similarly, transits are not independent from each other. Their mutual dependence comes from the level of occupation of the docks, defined by previous ship calls to harbor and from previous waiting times, as well as by the time series of operational condition of the navigation areas.

Due to this, equations (2) and (3) are only valid when the model includes all the mentioned aspects during the simulation, i.e.: temporal dependence of the agents, position of ships on time, docks occupation level and time series of the operational state of the navigation areas.

\section{IMPLEMENTATION}

The model was implemented for a study case, the expansion of the Bay of Cádiz Harbor, including only one failure mode: "bottom hitting" during transit.

For this, several previous studies were performed for the study of the climatic agents and for the study of the behaviour of the ships. Next, a brief description of the study case is presented. Then, a description of the previous studies is presented, along with the discussion of some specific aspects related with the way in which the results of the studies are included in the model. Finally, some aspects related with the numerical implementation of the model are discussed.

It should be noted that the model must be able to simulate several useful lives of the navigation areas within a reasonable time. Due to this, no numerical model for any of the physical phenomena involved is included in the simulation model. General procedure was to use numerical models to study physical phenomena previously to the implementation of the simulation model. Then, outputs of the numerical models were included into the simulation model as data bases, or were used to calibrate black-box models. This procedure assures that computation time remains short enough, and that the model can be run in a standard desktop computer.

\section{Case study: Bay of Cádiz Harbor expansion}

Bay of Cádiz Harbor is located on the south-west coast of Spain, at the Gulf of Cádiz, on the Atlantic Ocean. The harbour authority and the University of Granada are involved in an expansion 
project, consisting on a new specialized container terminal, with a lineal wharf of approximately 1000 $\mathrm{m}$ that allows the docking of ships of 6000 TEUs.

Design ship has $300 \mathrm{~m}$ length, $30 \mathrm{~m}$ beam and $13 \mathrm{~m}$ draft. In order to allow these ships to enter the harbor, a deepening and widening of the entrance channel is planned, along with a new manoeuvring area. Entrance channel has $5000 \mathrm{~m}$ length, and project depth is $16 \mathrm{~m}$ relative to the mean sea level.

Figure 2 shows location of the Bay of Cádiz Harbor, along with an outline of the expansion project.

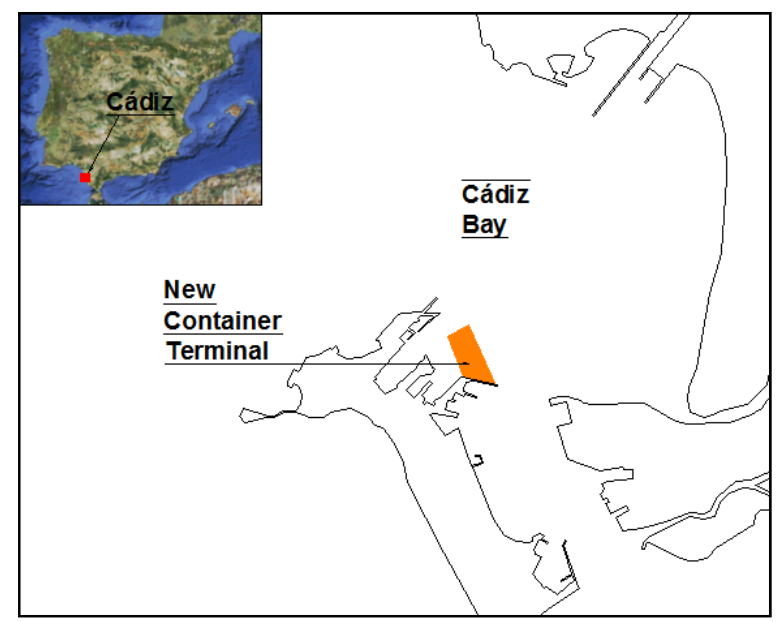

Figure 2. Location of the Bay of Cádiz Harbor and outline of the projected expansion.

The useful life of the Project, and its requirements in terms of safety and operationality, are estimated following Losada (2001), resulting in a useful life of 25 years, with a minimal operationality of $95 \%$ and a maximum overall failure probability during its useful life of $10 \%$.

\section{Climatic agents modeling}

First step for the implementation of the model was the study of the climatic agents. Main objective of this is: given a time series of sea states and astronomical tides, estimate total sea level, wave conditions and currents on each subset.

Wave propagation. Wave data available for the project consists on deep water wave height, period and direction obtained through hindcasting (Spain Port Authorities; Puertos del Estado de España, www.puertos.es). For wave propagation from deep waters to each subset, a data base is constructed. 500 sea states are defined with different significant wave heights, peak period and peak direction, and propagated with Oluca-SP (spectral parabolic model based on REF/DIF-S). This data base is used for propagation of wave series through multidimensional interpolation. The model uses the data base for the propagation of the available wave data; however it can be used for the propagation of any wave series that may be available on the future (measured, hindcasted or simulated).

Sea level. Sea level is assumed uniform on all subsets, and composed by astronomical (deterministic) and meteorological tides. Astronomical tide is obtained by means of the Tide Preview software, using tidal constituents of the Bay of Cádiz Harbor. Meteorological tide on Cádiz area follows a normal distribution, whose parameters depends on the significant wave height in deep waters (Grupo de Puertos y Costas, 2008). This way, astronomical tide and deep water wave height are inputs to the model, while meteorological tide is estimated into the model.

Tide generated currents. Two weeks of levels and currents measured on the existing entrance channel are used for the calibration of a 2D hydrodynamic numerical model of the Bay of Cádiz. Then, the bathymetry is modified in order to include the new channel layout and the new manoeuvring area, and three month of tides and currents are simulated.

The three months of numerically modelled currents are used to calibrate a Hammerstein-Wiener model (see e.g. Greblicki and Pawlak, 2003) for each subset of the navigation areas. These models are used as black-boxes into the simulation model, giving the current speed and direction at each time step as a function of present and past sea levels.

The use of black-boxes models allows for a good approximation of the agents that are generated from other agents (as is the case of currents generated by sea level variations), with a negligible computational cost. 
Time series simulation. One of the main sources of variability in the outputs of the model is the variability of the climatic agents. The model requires the input of time series of wave height, period and direction on deep waters, as well as speed and direction of the wind. Knowing this series, data bases and black-boxes are used into the model for the estimation of all needed agents at each subset.

Time series can be measured or hincasted. However, for taking into account climatic variability and mid and long term trends, it is necessary to simulate new time sires through Monte Carlo methods. There are several techniques available for multivariate time series simulation: translated Gaussian process (see e.g.: Borgman and Scheffner, 1991), multivariate ARMA models (see e.g.: Guedes Soares and Cunha, 2000). A summary of available techniques can be found at Monbet et al. (2007).

In this work results are obtained using a measured time series. At the moment work is being carried on in order to obtain results based on simulated time series.

\section{Use and exploitation agents modeling}

Main use and exploitation agent included on the model is the ship, which must enter into the harbor, load/unload cargo, and exit the harbor. Variability in this agent is given by: (a) time series of ships calling to harbor and the volume of cargo to be moved by each ship at the harbor, (b) the behaviour of the ship on plan view, and (c) the behaviour of the ship on elevation view. These three aspects are discussed next.

Time series of ships calling harbor. It is expected that the new container terminal serves a mean of one ship per day. Given that the exploitation of the terminal will be in hands of a third party, there is not a precise analysis of the expected fleet and cargo volumes.

For this work it is assumed that all ships will be similar to the design ship, with interarrival times given by an exponential distribution. The use of the exponential distribution for modelling interarrival times is widely used in the bibliography (see e.g.: Shabayek and Yeung, 2001, Pachakis and Kiremidjian, 2003).

Service time at docks can be treated as one of the parameters of the model and can be adjusted according to the available information when there is any. For example, knowing the volume of cargo to be moved by each ship and the efficiency and number of the cranes of the terminal, it would be possible to obtain service time from them. In this work no information is available. It is assumed that service time follows a uniform distribution, with times between 12 and 72 hours.

Plan view studies. A plan view study was performed with an autopilot model. The objective of the study was the definition of the climatic condition under which a transit can be performed without exceeding the channel way marks, and the number of tows needed for it. Given a climatic condition the response of the autopilot model is deterministic, as a consequence the plan view analysis is deterministic, i.e.: given the climatic conditions the autopilot model returns one of the two possible results: the ship succeed on performing the transit, or the transit fails because the ship exceeds the channel way marks.

18,000 entrance transits and 18,000 exit transit are simulated for each possible configuration of the tows. Simulations cover a wide range of possible climatic conditions: 50 sea stated, with directions from $\mathrm{NW}$ to $\mathrm{W}$, taken every $22.5^{\circ}$, wave height equal to 2,4 , and $6 \mathrm{~m}$, and periods equal to $7,9,11$, and $13 \mathrm{~s}, 9$ current states, including extreme ebb and flow tide condition, and winds from every direction, taken every $45^{\circ}$, with speeds between 6 and $22 \mathrm{~m} / \mathrm{s}$, taken every $4 \mathrm{~m} / \mathrm{s}$.

All these climatic conditions were studied with different number ( 3 to 5 ) and location of tows. Final result obtained from plan view analysis is a chart of climatic conditions under which transit can take place without the ship exceeding channel way marks. This result is included into the simulation model through the usage policy of the navigation areas.

Elevation view study. Defined a transit state, it is possible to estimate the probability of the ship hitting the bottom assuming that the amplitudes of the ship oscillation motions are independent and identically distributed, with a Rayleigh distribution:

$$
P_{f_{-} i}=1-\left(1-\exp \left\{-\frac{a^{2}}{2 m_{0}}\right\}\right)^{\frac{T_{i}}{2 \pi} \sqrt{\frac{m_{2}}{m_{0}}}}
$$

where $\boldsymbol{a}$ is the under keel clearance available for vertical oscillation of the ship, $\boldsymbol{m}_{\boldsymbol{0}}$ and $\boldsymbol{m}_{2}$ are zero and second order moments of the spectrum of the vertical movements of the ship (at bow or stern), and $\boldsymbol{T}_{\boldsymbol{i}}$ is the length of the transit state $\boldsymbol{i}$.

For estimating $\boldsymbol{a}$, the following is taken into account: 
1. Water level related factors: astronomical and meteorological tide (both stationary during a transit state).

2. Seabed related factors: channel depth and bathymetry inaccuracies (both stationary during the entire transit).

3. Ship related factors: ship draft, dynamic trim, heeling caused by wind, heeling caused by currents, heeling due to course alterations, clearance for safety and control of the ship's manoeuvrability (all taken as stationary during a transit state).

All these factors are calculated following recommendation ROM 3.1-99 (Puertos del Estado, 2007).

The spectrum of the vertical movements of the ship depends on: hull form and its mass distribution, ship speed and direction, as well as on waves, currents and sea levels. All those aspects can be taken into account by numerically modelling the behaviour of the ship, or using simplified expression included in Puertos del Estado (2007). In this work both approaches are used.

A 3D numerical model based on panel theory was used for the calculation of the transfer function from the wave spectrum to the ship vertical movement spectrum. Given a specific hull and mass distribution, transfer functions depends on water depth and ship speed. Model is applied for three different depths $(14,16$ and $18 \mathrm{~m}$ ) and for 8 ship speeds (from 0 to $3.5 \mathrm{~m} / \mathrm{s}$, taken every $0.5 \mathrm{~m} / \mathrm{s}$ ). Then, assuming an unidirectional TMA wave spectrum, with wave height $1 \mathrm{~m}$, coming from different directions, a data base of spectral moments $\boldsymbol{m}_{0}$ and $\boldsymbol{m}_{2}$ is constructed. This data base is included into the simulation model, where it is used to interpolate $\boldsymbol{m}_{0}$ and $\boldsymbol{m}_{2}$ at each subset, as a function of relative speed and direction of the ship, of the water depth, and of the direction and the peak period of the waves. Then, assuming linear theory, moments are scaled using the significant wave height. Knowing the moments, failure probability during a transit state is estimated by means of (4).

The methodology proposed in Puertos del Estado (2007) was also included in the model. This methodology provides the significant amplitude of the vertical movements of the ship. This amplitude is equivalent to $2 \sqrt{m_{0}}$. In order to use (4) with this estimation of $\boldsymbol{m}_{0}$ it is needed to assume the mean period of oscillation of the ship. In this work it was taken equal to the peak period of the wave spectrum. An alternative would have been the use of the characteristic oscillation period of the ship that is independent of the wave conditions.

\section{Subsets and transit states definitions}

Taking into account the results of the previous studies, four subsets were defined, as outlined on Figure 3. In the manoeuvring area (subset 4) the ship changes its direction continuously at an approximately constant speed. Because of that, subset 4 is subdivided during calculation in several subsets, for which it is possible to assume an approximately constant direction of the ship.

The length of the sea state is taken to be three hours. This is the time step of the available wave and wind data. Wave and wind variables are assumed statistically stationary during a sea state.

Astronomical tide is semidiurnal. Taking into account the tide amplitudes in Cádiz, it is concluded that the rate of change of the sea level can be of order $1 \mathrm{~cm} / \mathrm{min}$. Therefore it is assumed that the length of a sea level state is $10 \mathrm{~min}$. Within this time the sea level is assumed stationary.

Climatic state length is taken as the minimum length of the previously defined states, i.e.: $10 \mathrm{~min}$.

\section{Programming of the model}

Model is implemented on Simulink ${ }^{\circledR}$ Matlab ${ }^{\circledR}$ environment. For climatic agents transformation the data base of propagated waves and the black-box models for currents are included. For the estimation of the response of the ship on waves, the data base of zero and second order moments of the ship vertical displacement spectrum and the ROM 3.1-99 methodology are included.

Transformation of the climatic agents is made using a constant time step, equal to the length of the climatic state. The simulation of the transits is performed using discrete event programming. Transit state is estimated into the model. The change from one transit state to the next occurs when the ship enters into a new subset or when the climatic state changes. For every transit state failure probability is estimated through (4). 


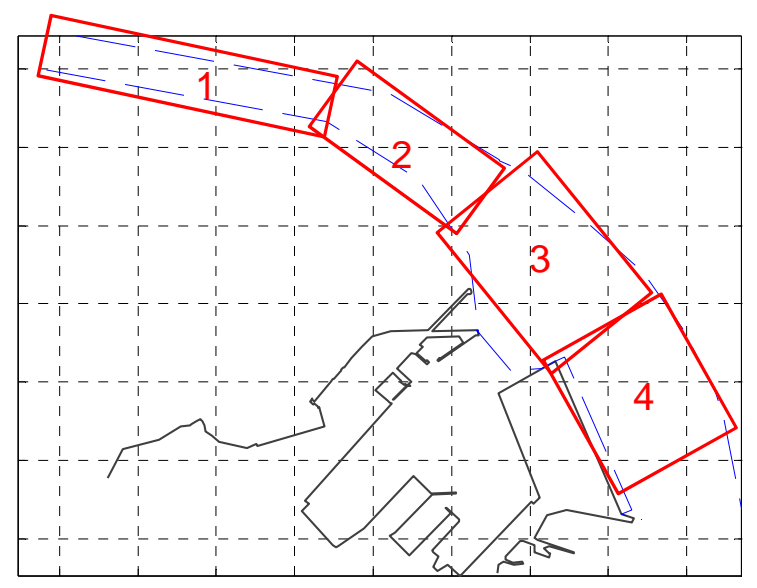

Figure 3. Outline of the subsets of the navigation areas.

\section{MODEL APPLICATION}

Here some of the results obtained with the model are presented. These results are divided on: verification and optimization of the design based on its safety, verification of the operationality, and decision support for management.

\section{Verification and optimization of the design based on safety estimates}

The model was used to study the safety of the system. For that, available climatic data was used.

Obtained results shows that the overall probability of a ship hitting the bottom during the useful life of the project is higher than maxima allowed, and that mayor failure probability occurred at subset one. Figure 4 shows failure probability on subset one during a transit, as a function of wave height and sea level. It is seen that mayor failure probabilities occurs for high wave height and low sea level.

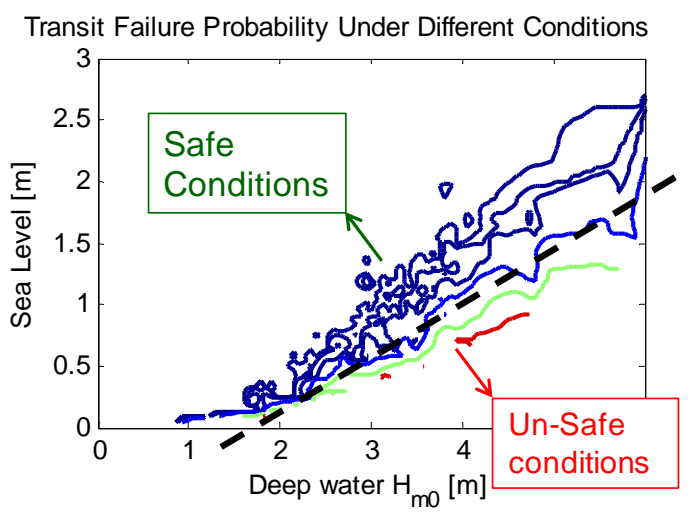

Figure 4. Probability of hitting the bottom on subset one during a transit, as a function of wave height and sea level conditions.

Possible measures to reduce the failure probability are the deepening of subset one and the modification of the usage policy of the navigation areas, in order to avoid "un-safe condition".

After testing several possible scenarios, it was decided to deepen subset one $50 \mathrm{~cm}$ and to impose that transits can only take place when $\mathrm{SWL}>\mathrm{Hs}-2 \mathrm{~m}$, for $\mathrm{Hs}>2 \mathrm{~m}$.

This way it is verified that the expected failure probability on 13 year (calculates performing several simulation using the available climatic agents and different simulates use and exploitation agents) is $0.02 \%$. This is low enough to say that overall failure probability in the useful life will not exceed the maximum allowed $(10 \%)$.

It must be noted that final estimation of the overall failure probability on the useful life must be carried out using simulated time series of the climatic agent. The length of the time series must be equal to the length of the useful life, and the number of simulations that must be performed should be estimated in order to obtain acceptable confidence intervals. 


\section{Operationality and performance verification}

Defined the new alternative, and verified that it fulfils pre-established safety requirements, it is important to verify if it fulfils operationality requirements as well.

Operationality is given by the model straightforward, and for the defined alternative is $96.7 \%$. This is higher than the minimum required for the project. Along with this, the model also provides information about waiting times and occupational level of the docks.

Figure 5 shows relative histograms of downtime length and total waiting times for entrance and exit transits. It is noted that downtime length approximately follows a uniform distribution between 3 and 40 hours, and that there are longer downtimes that corresponds to winter storms. In regards with the waiting times, it is noted that $60 \%$ of the ships did not have to wait (not included on the figure). However, there are a non negligible percentage of the transits that have excessively high waiting time. This is mainly due to the occupational level of the docks, that is too high for the available number of berths probably because of the distribution assumed for service times.

As it was stated for safety estimation, final operationality estimation must be done using simulated time series of climatic agents.
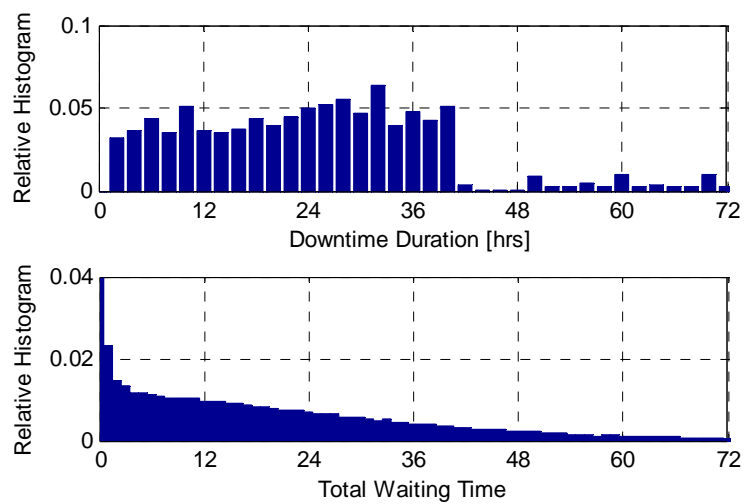

Figure 5. Relative histograms of the downtime duration and the waiting times, both in hours.

\section{Decision support for management}

A project alternative was defined by means of the geometry and the usage policy of the navigation areas that fulfils the required levels of safety and operationality. However, once the navigation areas becomes operative, it is necessary to assure that the defined usage policy is applied, otherwise failure probability could be higher than estimated. For this an operation chart for the navigation areas was developed (Figure 6). This chart defines operational conditions as a function of wave height, water level, wind speed, wind direction $\left(\theta_{\mathrm{W}}\right)$ and current speed $\left(\mathrm{U}_{\mathrm{C}}\right)$.

On the other hand, if the simulation model is implemented in the harbour as a real time or short term forecast tool, it would be feasible the estimation of the failure probability of each transit before the transit take place, and this could be used as a decision support tool for pilots to define whether a transit should take place or not.

\section{DISCUSSION}

Most resent standards and recommendations state that maritime work projects of high repercussion (economic, social and/or environmental) have to be verified by means of level III techniques (see e.g.: Losada, 2001). Accordingly, in recent years an increase in the risk based design of maritime works has been observed, which requires a precise estimation of failure probabilities.

Navigation areas are complex systems, where safety, operationality and service levels are interrelated. Direct Level III verification of these systems may be difficult to perform, and a model that simulates the evolution of the system with time is an alternative for perform such verification.

In this work the methodology employed for the implementation of a simulation model of the navigation areas of a container terminal was shown. The model integrates safety, operationality and performance. 


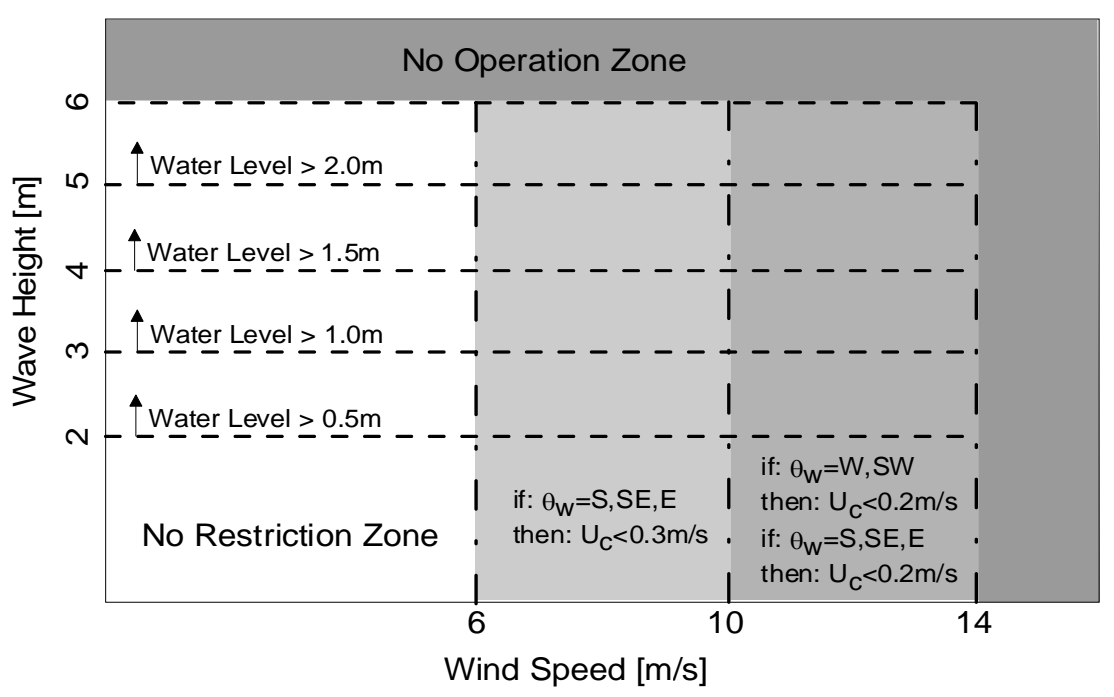

Figure 6. Operation chart for navigation areas.

In regards with the applicability of the model, it was presented an example of its use in the design process (the definition of the geometry and the usage policy of navigation areas), so that preestablished safety and operationality requirements are fulfilled.

So far the model was implemented for a container terminal, with a fleet composed only by the design ship, and including one failure mode (bottom hitting during transit). However, the methodology applied can be easily extended to other terminals, more complex fleets and other failure modes.

It should be noted that the estimation of the failure probability and the operationality during the useful life must be done simulating several useful lives of the system. This requires time series of climatic agents that can be measured time series, when available, or simulated time series.

Computation times needed for the application of the model are of the order of five simulated years per minute using a standard desktop computer. Although this time could increase if more complex fleets and terminals are included into the model, it is believed that computation times would remind low enough, so that the model could be used during the design process.

Main research lines still opened related with this work are the implementation of the model as a real time decision support tool, and the development of a formal optimization procedure that can be used with the model, since so far optimization of the projects is based on the evaluation of several different scenarios.

\section{CONCLUSIONS}

In this work a new model was developed for a container terminal, and it was shown that by including on the model safety, operational and performance aspects, it is possible to perform an integral verification of the system, estimating the overall failure probability during the useful life of the navigation areas along with its operationality and the quality of the service provided.

The implementation of the model does not require a complex theoretical development, as long as the definition of the subsets and the transits states is performed as discussed in this work and in Losada et al. (2009). It does require an important amount of previous works, equivalent to that needed other type of probabilistic verifications. This includes climatic agent studies and ship response studies.

Special attention was paid during implementation to avoid the inclusion of computational demanding numerical models into the simulation model. In this way, the simulation model is not very demanding in terms of computation resources, and can be used for the evaluation of several different scenarios in order to optimize a project.

\section{ACKNOWLEDGMENTS}

Authors would like thank Bay of Cádiz Harbor authority for its collaboration and partial founding, trough "Estudios para la Ampliación del Puerto de la Bahía de Cádiz" project. The authors are also indebted to the Ministry of Fomento, Spain, for partial founding through projects CIT-460000-2009-21 and 53/08 (orden FOM/3864/2008). The first author thanks the Ministry of Education of Spain for financial support through "FPU” scholarship program (Ref. Number: AP2008-03235). 


\section{REFERENCES}

Bierwirth, C., and Meisel, F., 2010. A survey of berth allocation and quay crane scheduling problems in container terminals. European Journal of Operational Research, 202, 615-627.

Borgman, L.E. and Scheffner, N.W. 1991 The simulation of time sequences of wave height, period and direction. Tech. Rep., Coastal Engineering Research Center, U.S. Army Engineer Waterways Station.

Briggs, M.J., Borgman, L.E. and Bratteland, E. 2003 Probability assessment for deep-draft navigation channel design. Coastal Engineering, 48, 29-50.

Greblicki, W. and Pawlak, M., 2003. Nonparametric System Identification. Cambridge University Press. ISBN 0521868041

Grupo de Puertos y Costas, 2008 Estudio de alternativas para la ampliación de la dársena portuaria de Cádiz. Tech. Rep. Universidad de Granada (in Spanish)

Guedes Soares, C. and Cunha, C. 2000 Bivariate autoregressive models for the time series of significant wave height and mean period. Coastal Engineering, 40, 313-327.

Losada, M.A., 2001 ROM 0.0 General procedure and requirements in the design of harbor and maritime structures. Part I. Puertos del Estado, editor. ISBN 84-88975-30-9

Losada, M.A., Baquerizo, A., Ortega-Sánchez, M., Santiago, J.M. and Sánchez-Badorrey, E., 2009. Socioeconomic and Environmental Risk in Coastal and Ocean Engineering, on Handbook of Coastal and Ocean Engineering, Kim, Y.C. Ed. ISBN 978-981-281-929-1.

Monbet, V., Alliot, P. and Prevosto, M. 2007 Survey of stochastic models for wind and sea state time series. Probabilistic Engineering Mechanics, 22, 113-126.

Pachakis, D. and Kiremidjian, A.S. 2003 Ship Traffic Modeling Methodology for Ports. Journal of Waterway, Port, Coastal, and Ocean Engineering, 129 (5), 193-202.

Puertos del Estado (Ed.), 2007. ROM 3.1-99 Recommendations for the Design of the Maritime Configuration of Ports, Channels and Harbour Basins. ISBN 84-88975-39-2

Quy, N., Vrijling, J., van Gelder, P., 2008. Risk- and simulation-based optimization of channel depths: Entrance channel of cam pha port. Simulation, 84(1) 41-56.

Shabayek, A. and Yeung, W., 2001. Effect of seasonal factors on performance of containers terminals. Journal of Waterway, Port, Coastal and Ocean Engineering, 127(3), 135-140.

Spencer, J.M.A., Bowers, E.C., and Lean, G.H. 1990. Safe underkeel allowances for vessels in navigation channels. Proceeding of the 22nd International Coastal Engineering Conference. Delft, The Netherland. ISBN 0-87262-776-4 